\title{
CIBERACOSO Y RECURSOS PERSONALES EN ADOLESCENTES: LAS COMPETEN- CIAS EMOCIONALES Y LA GRATITUD COMO FACTORES DE PROTECCIÓN ANTE LAS CONDUCTAS DE CIBERAGRESIÓN
}

\author{
María Teresa Chamizo-Nieto y Lourdes Rey \\ Universidad de Málaga, España
}

\begin{abstract}
Resumen
Desde los últimos años se ha observado un aumento de conductas de ciberacoso que conlleva graves consecuencias negativas en los adolescentes implicados. Diversos estudios han analizado qué factores de riesgo y de protección influyen en estas situaciones, así como su repercusión sobre el bienestar de los adolescentes. Dos recursos personales que han evidenciado tener efectos protectores sobre el ajuste psicológico y la salud de los adolescentes son la inteligencia emocional (IE) y la gratitud. Sin embargo, son pocos los estudios que han examinado ambas variables en situaciones de ciberacoso. Por ello, el principal objetivo de esta investigación consistió en examinar el papel conjunto de ambos factores en el ciberacoso. Concretamente, se hipotetizó un efecto de interacción IE $\mathrm{x}$ gratitud para predecir la ciberagresión. Una muestra de 770 adolescentes españoles entre 12 y 14 años $(54,4 \%$ mujeres) cumplimentaron diferentes cuestionarios que evaluaban la IE (WLEIS-S), la gratitud (GQ-5) y el ciberacoso (ECIP-Q). Los resultados reflejaron correlaciones significativas en la dirección esperada entre las variables. Asimismo, la IE moderó el efecto principal de la gratitud sobre la ciberagresión. Estos resultados aportan evidencias sobre el papel protector de ambos recursos en situaciones de ciberacoso. Se comentan las implicaciones teóricas y prácticas de los hallazgos obtenidos.
\end{abstract}

Palabras clave: Inteligencia emocional, Gratitud, Ciberacoso, Factores protectores, Recursos personales, Adolescentes

\begin{abstract}
Since the last years, cyber-aggressive behaviours have increasing, having severe negative consequences on involved adolescents. Different studies have analysed which factors (protective and risk) have influence on these situations, as well as their impact on adolescents' well-being. Emotional intelligence (EI) and gratitude are two personal resources which have provided evidence about their protective effects on adolescents' psychological adjustment and health. Nevertheless, there are few studies which have examined both joint variables in cyberbullying context. Thus, the principal objective of this research was to examine the role of both factors in cyberbullying. Specifically, an interaction effect EI x gratitude was hypothesized to predict cyberbullying. A sample of 770 Spanish adolescents between 12 and 14 years (54.4\% females) filled out some questionnaires measuring EI (WLEIS-S), gratitude (GQ5) and cyberbullying (ECIP-Q). Outcomes showed expected significative correlations among variables. Likewise, EI moderated the principal effect of gratitude on cyber-aggression. These findings provide evidences about the protective role of both resources in cyberbullying situations. Theoretical and practical implications of findings obtained are discussed.
\end{abstract}

Keywords: Emotional intelligence, Gratitude, Cyberbullying, Protective factors, Personal resources, Adolescents

Correspondencia.

\author{
María Teresa Chamizo Nieto \\ mtchamizo@uma.es \\ Universidad de Málaga. España
}




\section{Introducción}

En las últimas décadas, gracias a la creación de dispositivos electrónicos (p.ej., los teléfonos inteligentes o smartphone) y la creación de diferentes redes sociales (como Facebook o Instagram), se ha producido un auge de las nuevas tecnologías, incorporando importantes cambios en la manera de comunicarnos y relacionarnos con otras personas. Uno de los aspectos negativos que conlleva el uso inadecuado de estas nuevas tecnologías es la aparición del fenómeno de ciberacoso o ciberbullying (Sanjuán, 2019).

\section{Ciberacoso}

El ciberacoso es una conducta violenta donde se realiza un daño de manera intencionada y repetitiva hacia otras personas mediante la utilización de dispositivos electrónicos o a través de los medios online (Smith et al., 2008), caracterizándose por una rápida difusión, por la posibilidad del anonimato por parte del agresor y la dificultad de las víctimas para poder defenderse y desconectar del medio virtual (Antoniadou y Kokkinos, 2015; Tokunaga, 2010). Este fenómeno está presente en multitud de países, existiendo diferencias en los datos de prevalencia (p.ej., LozanoBlasco et al., 2020). Camerini et al. (2020) han revisado 27 estudios longitudinales encontrando que la presencia de ciberacoso oscila desde un $5,3 \%$ hasta un $66,2 \%$ entre los estudios analizados, dependiendo de los instrumentos de medida que se han utilizado y el periodo de tiempo considerado. En España, una investigación realizada recientemente señala que uno de cada cinco adolescentes entre $11 \mathrm{y}$ 18 años está involucrado en ciberacoso (Calmaestra et al., 2020).

Literatura previa ha evidenciado el impacto negativo en la salud, el bienestar y el ajuste psicológico de los adolescentes implicados en esta problemática. Tanto las cibervíctimas como los ciberagresores presentan mayores niveles de estrés y mayor número de problemas emocionales y de conducta (Garaigordobil y Machimbarrena, 2019), como mayor sintomatología depresiva y ansiedad social, niveles más bajos de satisfacción vital (Estévez et al., 2019), y un mayor riesgo de intentos de suicidio (Hinduja y Patchin, 2019) que los adolescentes no implicados. Específicamente, entre los ciberagresores se encuentran bajos niveles de amabilidad, responsabilidad y empatía (Garaigordobil, 2019; Segura et al., 2020), mostrando también, menor regulación de las emociones (Cañas et al., 2020; Segura et al., 2020). En contraposición, presentan mayor sentimiento de soledad (Cañas et al., 2020), niveles más altos de estrés en la escuela y síntomas de ansiedad, mayores problemas de atención e hiperactividad (Garaigordobil y Machimbarrena, 2019) y más comportamientos violentos (Garaigordobil y Machimbarrena, 2019; Iranzo et al., 2019).
Teniendo en cuenta los graves efectos negativos derivados del ciberacoso sobre el funcionamiento social, personal y familiar de los adolescentes implicados, diferentes investigaciones se han centrado en indagar cuáles son los factores de riesgo que predicen la implicación en el ciberacoso, así como qué recursos y factores pueden proteger, previniendo y reduciendo esta problemática y el impacto negativo derivado de ella (Camerini et al., 2020; Kowalski et al., 2019; Zych et al., 2019). Entre los factores de riesgo que predicen las conductas de ciberagresión en adolescentes se han encontrado, por ejemplo, niveles altos de agresión, bajos en autocontrol y menor capacidad de empatía (Garaigordobil, 2019; You y Lim, 2016), así como un menor desarrollo o conciencia de las habilidades emocionales, como la regulación de las emociones (Baroncelli y Ciucci, 2014; Garaigordobil, 2019; Kokkinos y Voulgaridou, 2017). Por otro lado, la literatura ha analizado, también, diversos recursos y factores sociales, personales y familiares que pueden influir protegiendo y reduciendo la probabilidades de llegar a convertirse en un ciberagresor (p.ej., Kowalski et al., 2019; Quintana-Orts y Rey, 2018). Entre los recursos personales que han mostrado efectos beneficiosos para el ajuste psicológico de los adolescentes, la mejora en las relaciones interpersonales junto a la disminución de comportamientos agresivos, se encuentran la gratitud y la inteligencia emocional (Carbonell y Cerezo, 2019; Deng et al., 2019).

\section{Gratitud}

En numerosas investigaciones la gratitud ha mostrado efectos beneficiosos sobre el ajuste psicológico (p.ej., Deichert et al., 2019) y la salud física (ver Lavelock et al., 2016, para una revisión). Este recurso personal resalta en su definición dos aspectos: por un lado, se refiere a una disposición a ser consciente de la amabilidad y las buenas acciones de los demás, y a responder a ellas (McCullough et al., 2002). Por otro lado, hace referencia a un estilo u orientación vital en la que las personas aprecian las cosas buenas o positivas que existen en la vida (Wood et al., 2010). Entre los aspectos positivos derivados de ser agradecido y expresar gratitud a los demás se han encontrado, por ejemplo, un incremento de la empatía (Chen et al., 2020; DeWall et al., 2012), la autoestima (Lin, 2015), el bienestar subjetivo (Deng et al., 2019; Jans-Beken et al., 2018) y la satisfacción vital (Guse et al., 2017). También, diversas investigaciones han obtenido hallazgos sobre el papel de la gratitud para promover la generosidad, los comportamientos prosociales y la valoración social hacia los demás, disminuyendo los niveles de agresión, los comportamientos antisociales y las conductas denigrantes y de agresión física hacia los otros (Bono et al., 2019; Cho y Fast, 2012; Deng et al., 2019; DeWall et al., 2012; Yost-Dubrow y Dunham, 2018; You et al., 2020). 
Aunque existen algunos estudios recientes que arrojan evidencias sobre el papel protector de la gratitud para reducir las actitudes y los comportamientos agresivos en situaciones de violencia entre iguales (Chen et al., 2020; GarcíaVázquez et al., 2020), en el contexto del ciberacoso, el papel de la gratitud apenas ha sido examinado.

\section{Inteligencia Emocional}

Otro factor que ha mostrado resultados beneficiosos en la promoción de la salud y el ajuste psicológico de los adolescentes es la inteligencia emocional (IE). Esta se define como la habilidad mental que permite la identificación, percepción, comprensión y regulación de las emociones propias y de los demás, así como su uso y actuación sobre las mismas (Mayer et al., 2016; Mayer y Salovey, 1997). El desarrollo de las habilidades emocionales está relacionado con resultados positivos sobre el individuo como mayor satisfacción vital (Extremera y Rey, 2016) y bienestar subjetivo (Geng, 2018), un mejor ajuste psicológico y funcionamiento social (Cejudo et al., 2018), y el empleo de estrategias de afrontamiento más productivas para hacer frente a las situaciones estresantes cotidianas (p.ej., mediante la puesta en marcha de una solución activa o el mantenimiento de una actitud positiva ante la situación) (Morales Rodríguez, 2017).

En el ámbito de la violencia, los resultados de investigaciones previas sugieren que un buen desarrollo de la IE está relacionado con una menor conducta agresiva (Morales Rodríguez, 2017). En este sentido se ha observado que, aquellos adolescentes con mayores habilidades de IE presentan una mejor resolución de conflictos sociales y una disminución de las conductas agresivas (Garaigordobil y Peña-Sarrionandia, 2015). Así mismo, aquellas personas con un menor desarrollo de las habilidades emocionales informan de mayores niveles de odio, agresión y hostilidad (Inglés et al., 2014) y una mayor realización de comportamientos agresivos (ver García-Sancho et al., 2014, para una revisión).

En el contexto de ciberacoso específicamente, los hallazgos obtenidos tras la realización de programas para desarrollar la IE en adolescentes, como el PREDEMA (Schoeps et al., 2018) o el CIE (Carbonell y Cerezo, 2019), sugieren, igualmente, que un entrenamiento y desarrollo de las competencias emocionales puede disminuir el ciberacoso (Carbonell y Cerezo, 2019; Schoeps et al., 2018) y fomentar el bienestar subjetivo en esta población (Schoeps et al., 2018). Por tanto, un incremento de las habilidades emocionales, como la comprensión o la regulación emocional en los adolescentes, puede reducir las probabilidades de que lleguen a convertirse en ciberagresores (Martínez-Monteagudo et al., 2019; Quintana-Orts y Rey, 2018).

\section{Acción conjunta de la inteligencia emocional y la grati- tud sobre la ciberagresión}

Los hallazgos de las investigaciones previas muestran evidencias de que la IE y la gratitud, como variables independientes, pueden disminuir la realización de comportamientos agresivos y mejorar el ajuste psicológico y las relaciones en los adolescentes. Considerando la relación entre estos recursos personales y sus consecuencias beneficiosas, podría hipotetizarse una potencial interacción de ambos recursos que protejan contra la ciberagresión. A pesar de que existe poco conocimiento acerca de la relación y acción conjunta de la IE y la gratitud sobre el bienestar y la salud, parece que ambos factores, como variables independientes, podrían interaccionar y complementar sus efectos positivos en diferentes áreas y contextos (p.ej., social, personal, familiar o académico). Sin embargo, hasta la fecha, no se ha encontrado un estudio que haya explorado esta acción conjunta de ambos recursos personales en el contexto de ciberacoso. Por tanto, el propósito principal de este estudio fue examinar la posible interacción de la IE y la gratitud sobre las conductas de ciberagresión.

\section{Objetivos del presente estudio}

Este estudio planteó tres objetivos para profundizar e incrementar el conocimiento del papel de la IE y la gratitud en situaciones de ciberacoso. El primer objetivo consistió en examinar las asociaciones entre la IE, la gratitud y la ciberagresión. De acuerdo a la literatura previa (p.ej., Chen et al., 2020; Preś et al., 2020; Yudes et al., 2020), esperamos hallar correlaciones significativas y negativas entre la ciberagresión y ambos recursos, y positivas entre la IE y la gratitud. Con el segundo objetivo se analizan las relaciones de ambos recursos con la ciberagresión. Considerando hallazgos previos sobre el papel protector de la IE en el ciberacoso (p.ej., Carbonell y Cerezo, 2019; Yudes et al., 2020) y de la gratitud sobre las actitudes y los comportamientos agresivos (p.ej., Chen et al., 2020; García-Vázquez et al., 2020), se espera encontrar que ambos recursos expliquen, de manera independiente, una disminución de la conducta ciberagresiva. En el tercer y último objetivo, se pretende explorar el papel moderador de la IE en la relación entre gratitud y ciberagresión. Teniendo en cuenta que ambos recursos, individualmente, pueden explicar una reducción de los comportamientos agresivos (p.ej., Carbonell y Cerezo, 2019; Deng et al., 2019), y las evidencias de algunos estudios sobre las relaciones positivas que se establecen entre la IE y la gratitud (p.ej., Preś et al., 2020; Salve y Lavalekar, 2017), esperamos encontrar un efecto de interacción de ambos recursos en la predicción de la ciberagresión. 


\section{Método}

\section{Participantes}

Participaron 770 adolescentes (351 varones y 419 mujeres) entre 12 y 14 años $\left(\mathrm{M}_{\text {edad }}=12,997, D T=0,79\right)$ pertenecientes a cinco centros educativos de la provincia de Málaga. El nivel de estudios de la muestra abarca los cuatro cursos de educación secundaria obligatoria (ESO). El 97,8\% de los adolescentes tenían nacionalidad española.

\section{Instrumentos}

Previo a la cumplimentación de los cuestionarios se les solicitó a los participantes los siguientes datos sociodemográficos: sexo, edad, nacionalidad y curso escolar.

European Cyberbullying Intervention Project Questionnaire (ECIP-Q; Del Rey et al., 2015). Se utilizó la subescala de ciberagresión de la versión española de Ortega-Ruiz et al. (2016) que mostró una buena fiabilidad. Esta subescala mide mediante 11 ítems la frecuencia en la que se han realizado conductas de ciberagresión durante los últimos dos meses. Cada ítem se responde mediante una escala tipo Likert de cinco opciones de respuesta que van desde 0 (no) hasta 4 (sí, más de una vez por semana). Un ejemplo de ítem sería "He pirateado la cuenta de correo de alguien y he robado su información personal (email o red social)". En el presente estudio se obtuvo una fiabilidad de la subescala de 0,81 .

Wong and Law' Emotional Intelligence Scale (WLEIS; Wong y Law, 2002). Se utilizó la versión española de Extremera et al. (2019) que obtuvo una adecuada consistencia interna. Esta escala evalúa cuatro aspectos de la IE mediante 16 ítems: evaluación de las propias emociones (p.ej., "Tengo una buena comprensión de mis propias emociones"), evaluación de las emociones de los demás (p.ej., "Soy sensible a los sentimientos y emociones de los demás"), uso de las emociones (p.ej., "Siempre me digo a mí mismo que soy una persona competente"), y regulación de las emociones (p.ej., "Me puedo calmar fácilmente cuando me siento enfadado"). Cada ítem se responde mediante una escala tipo Likert de siete opciones de respuesta, desde 1 (completamente en desacuerdo) a 7 (completamente de acuerdo). Esta escala permite obtener una puntuación total de IE y una puntuación por dimensiones. Para este estudio, se ha considerado la puntuación total, siendo el alfa de Cronbach de la escala total en este estudio de 0,88 .

Gratitude Questionnaire (GQ; McCullough et al., 2002). Se utilizó la versión española para adolescentes de Rey et al. (2018) compuesta por cinco ítems y que presentó buenas propiedades psicométricas. Un ejemplo de ítem sería "Si tuviera que hacer una lista de todo por lo que me siento agradecido/a, sería una lista muy larga". Este cuestionario evalúa la disposición a estar agradecido, respondiendo cada ítem mediante una escala tipo Likert de siete opciones de respuesta siendo 1 (completamente en desacuerdo) y 7 (completamente de acuerdo). La fiabilidad del cuestionario obtenida en este estudio fue de 0,77 .

\section{Procedimiento}

La realización del presente estudio contó con la aprobación del Comité Ético de la Universidad de Málaga (62-2016-H) y estuvo de acuerdo con la Declaración de Helsinki (2013). El procedimiento consistió en la cumplimentación de una batería de cuestionarios, como parte de un proyecto más amplio, de manera anónima y voluntaria por parte de estudiantes adolescentes. Para ello, en primer lugar, se contactó con diferentes centros educativos de la provincia de Málaga, informando a los directores y miembros del equipo educativo sobre los objetivos y la metodología que se seguiría en la investigación. En aquellos centros que aceptaron participar, tanto los directores de los centros como los padres o tutores legales de los alumnos, firmaron un consentimiento informado de participación. En cuatro de los cincos centros educativos, el consentimiento fue activo (mediante la firma para su participación), mientras que en uno de ellos el consentimiento fue pasivo (no negaron explícitamente su participación). La cumplimentación de los cuestionarios se realizó durante el horario de tutoría. Durante el desarrollo de la misma, estuvieron presente dos investigadores que dieron indicaciones y resolvieron cualquier duda que los participantes tuvieran, así como un docente del centro educativo. En todo momento, se garantizó la confidencialidad de los datos y la voluntariedad de la participación en el estudio.

\section{Análisis de datos}

Se utilizaron el programa SPSS 24.0 y la macro de PROCESS (Hayes, 2018) para llevar a cabo los análisis de datos. Previo a los análisis se procedió a realizar la imputación de todos los valores perdidos mediante el método EM (expectation-maximization) con el programa SPSS 24.0. A continuación, se calcularon la media, desviación típica y consistencia interna de las tres variables de estudio (IE, gratitud y ciberagresión). Posteriormente, se realizó el análisis de correlación de Pearson entre las tres variables. Por último, para examinar el papel moderador de la IE en la relación entre gratitud y ciberagresión se empleó la macro de PROCESS (modelo 1; Hayes, 2018).

\section{Resultados}

\section{Estadísticos descriptivos, consistencias internas y corre- laciones}

La Tabla 1 muestra las consistencias internas de cada una de las variables del estudio, así como su media, desviación típica y las correlaciones de Pearson. Tal y como se esperaba, se encontró una correlación positiva y significativa entre la IE y la gratitud, mientras que la ciberagresión correlacionó negativa y significativamente con ambos recursos personales. 
Tabla 1. Estadísticos descriptivos, consistencias internas y correlacio-
nes

\begin{tabular}{lcccccc}
\hline & $\boldsymbol{\alpha}$ & $\mathbf{M}$ & $\mathbf{D T}$ & $\mathbf{1}$ & $\mathbf{2}$ & $\mathbf{3}$ \\
& & & & & & \\
\hline Ciberagresión & 0,81 & 0,14 & 0,29 & - & \\
$\begin{array}{l}\text { Inteligencia } \\
\text { emocional }\end{array}$ & 0,88 & 4,88 & 1,05 & $-0,16^{* *}$ & - & \\
$\begin{array}{l}\text { Gratitud } \\
\text { M }\end{array}$ & 0,77 & 5,70 & 1,16 & $-0,17^{* *}$ & $0,48^{* *}$ & - \\
\hline
\end{tabular}

Nota. $M=$ Media, $D T=$ Desviación Típica; $* * p<0,01$.

\section{Análisis de Moderación}

Para examinar el efecto moderador de la IE en la relación entre gratitud y ciberagresión, se utilizó el modelo 1 de la macro de PROCESS de Hayes (2018). En este modelo, el sexo fue introducido como covariable. Los resultados mostraron un efecto significativo de la IE $(\beta=-0,025, p<0,05)$ y la gratitud $(\beta=-0,023, p<$ $0,05)$ sobre la ciberagresión. Así mismo, se encontró un efecto significativo de la interacción IE x Gratitud sobre la ciberagresión $(\beta=0,016,95 \%$ IC: 0,001 a $0,030, t=2,131, p<0,05$ ) (ver Tabla 2 ). Tal y como se muestra en la Figura 1, la relación negativa entre gratitud y ciberagresión fue significativa a niveles bajos de IE $(\beta=-0,039,95 \%$ IC: $-0,060$ a $-0,017$, $t=-3,505, p<0,001)$, mientras que no lo fue a niveles altos de IE $(\beta=-0,006,95 \%$ IC: $-0,035$ a 0,023 , $t=-0,382, p=0,703)$.

Tabla 2.

Efecto moderador de la inteligencia emocional sobre la gratitud

\begin{tabular}{|c|c|c|c|c|c|c|}
\hline & \multirow[t]{2}{*}{$\beta$} & \multirow[t]{2}{*}{$\mathbf{S E}$} & \multirow[t]{2}{*}{$\mathbf{R}^{2}$} & \multirow[t]{2}{*}{$\Delta R^{2}$} & \multicolumn{2}{|c|}{$95 \%$ IC } \\
\hline & & & & & Bajo & Alto \\
\hline Ciberagresión & & & $0,043 * * *$ & & & \\
\hline Constante & $0,171 * * *$ & 0,033 & & & 0,105 & 0,236 \\
\hline Sexo & $-0,028$ & 0,020 & & & $-0,068$ & 0,012 \\
\hline IE & $-0,025^{*}$ & 0,011 & & & $-0,047$ & $-0,004$ \\
\hline Gratitud & $-0,023 *$ & 0,010 & & & $-0,043$ & $-0,003$ \\
\hline IE x Gratitud & $0,016^{*}$ & 0,007 & & $0,006^{*}$ & 0,001 & 0,030 \\
\hline
\end{tabular}

Nota. IE $=$ Inteligencia Emocional; IC $=$ Intervalo de confianza; $* p<0,05 ; * * * p<0,001$.

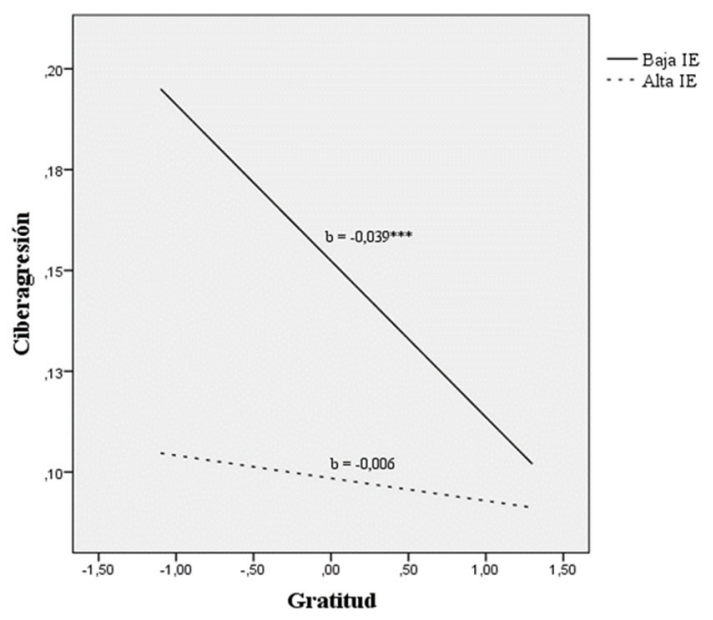

Figura 1.

El papel moderador de la inteligencia emocional (IE) en la relación entre la gratitud y la ciberagresión.

$* * * p<0,001$. 


\section{Discusión}

El propósito de este estudio fue analizar el papel protector conjunto de la IE y la gratitud en contextos de ciberacoso. Hasta la fecha, este es el primer estudio que examina el efecto de interacción de ambos recursos personales para predecir la ciberagresión en una muestra de adolescentes españoles de 12 a 14 años. Con el primer objetivo se pretendió examinar las correlaciones entre la gratitud, la IE y la ciberagresión. De acuerdo a las evidencias de la literatura previa (Cañas et al., 2019; Chen et al., 2020; GarcíaVázquez et al., 2020; Geng, 2018; Preś et al., 2020; Yudes et al., 2020), los resultados de este estudio mostraron correlaciones significativas entre las tres variables en las direcciones esperadas. Así, se ha observado que aquellos adolescentes con un mejor desarrollo de las competencias emocionales muestran una mayor conciencia sobre las cosas positivas que experimentan en su vida cotidiana. Además, se ha encontrado asociaciones entre una disminución en la frecuencia de realizar conductas y comportamientos agresivos o dañinos a través del ciberespacio en aquellos adolescentes que reflejan niveles altos a la hora de comprender y regular las emociones propias y ajenas, y una mayor disposición a la hora de sentir y expresar gratitud.

En relación al papel ejercido por ambos recursos personales sobre la ciberagresión, los hallazgos obtenidos sugieren, en primer lugar, que el desarrollo y práctica de la gratitud en los adolescentes conduce a una menor realización de conductas agresivas, como los insultos, la suplantación de la identidad en los perfiles sociales o la exclusión de grupos virtuales, entre otras acciones. Los resultados de este estudio están en consonancia con investigaciones previas donde se ha encontrado que las personas agradecidas, disminuyen los comportamientos agresivos en situaciones de acoso tradicional (García-Vázquez et al., 2020) y muestran una menor actitud para llevar a cabo conductas de ciberacoso (Chen et al., 2020). Por ello, se sugiere que los beneficios de ser una persona agradecida en las relaciones interpersonales que tienen lugar en contextos físicos compartidos (p.ej., Howells, 2014), se mantienen cuando se habla del ciberespacio, donde nos relacionamos unos con otros sin la necesidad de compartir un mismo espacio físico y donde la identidad de las personas pueden diluirse fácilmente o cambiarse. Esto puede deberse a que prestar atención y responder a las buenas acciones que recibimos de otras personas, facilita el proceso de ponernos en el lugar de los demás, incrementa el hecho de que los valoremos socialmente, enriqueciendo y fortaleciendo los lazos afectivos, y disminuyendo la probabilidad de provocar algún daño intencionado a otras personas (Algoe et al., 2013; Chen et al., 2020; Cho y Fast, 2012; DeWall et al., 2012). Asimismo, dado que recibir y expresar gratitud en los intercambios personales mejora la manera en la que nos comunicamos, y repercute en una mayor satisfacción en las relaciones (Howells et al., 2017), se podría decir que ser agradecido puede facilitar la resolución de conflictos de una manera más asertiva y prevenir la realización de comportamientos ciberagresivos.

En segundo lugar, con este estudio se corroboran evidencias previas sobre el papel protector de la IE sobre las conductas de ciberagresión (p.ej., Martínez-Monteagudo et al., 2019). Un buen desarrollo de las competencias emocionales permite un afrontamiento más adaptativo y eficaz de las situaciones estresantes diarias y mejora el funcionamiento psicológico y social de los adolescentes (Cejudo et al., 2018; Morales Rodríguez, 2017). Uno de los factores de riesgo a la hora de realizar conductas de ciberagresión es tener un déficit en la regulación emocional (p.ej., Garaigordobil, 2019). Por tanto, entrenar y desarrollar buenas habilidades emocionales, como la identificación, comprensión o regulación emocional, podría ayudarles a afrontar adecuadamente las situaciones estresantes y difíciles, disminuyendo sus niveles de estrés y las respuestas agresivas (p.ej., Garaigordobil y Machimbarrena, 2019). Todo ello podría repercutir en una disminución de la probabilidad de que los adolescentes lleguen a convertirse en ciberagresores.

Por último, con respecto a la acción conjunta de la gratitud y la IE en ciberagresión, los análisis de moderación revelaron un efecto de interacción de ambos recursos para predecir la ciberagresión. Tal y como se puede observar en la Figura 1, aquellos adolescentes que presentan bajos niveles de gratitud y de IE son los que muestran una mayor probabilidad de llevar a cabo conductas de ciberacoso. Además, los adolescentes con altos niveles de competencias emocionales mostraron una menor probabilidad de implicarse en comportamientos ciberagresivos, independientemente de sus niveles en gratitud. La relación significativa entre la gratitud y la ciberagresión encontrada únicamente a niveles bajos de IE, sugiere, por una parte, la importancia de tener un buen desarrollo de habilidades emocionales para disminuir las conductas violentas a través de las nuevas tecnologías $\mathrm{y}$, por otra parte, que tener una mayor conciencia sobre los aspectos positivos del mundo reduce el riesgo de ciberacoso, aunque los adolescentes presenten bajos niveles de IE. Parece ser, por tanto, que la ausencia o el bajo nivel de desarrollo de ambos recursos puede suponer un riesgo para la realización de conductas ciberagresivas por parte de los adolescentes, mientras que el desarrollo de estos recursos personales puede reducir la probabilidad de aparición de dichas conductas. De esta manera, se sugiere que, aunque es importante desarrollar unas buenas competencias emocionales que protejan y prevengan de las situaciones de ciberacoso, el desarrollo de la gratitud podría ser un factor añadido que compense la falta de dichas habilidades emocionales en los adolescentes. 


\section{Limitaciones}

Este estudio no está exento de limitaciones que habría que tener en cuenta de cara a la generalización de los resultados. En primer lugar, la muestra estuvo compuesta de adolescentes sanos entre 12 y 14 años de una zona geográfica concreta de España. En segundo lugar, al desarrollarse el estudio en un corte transversal, hay que tener precaución a la hora de realizar inferencias, puesto que este tipo de diseños no permite establecer relaciones causales. Por todo ello, sería interesante poder realizar futuras investigaciones longitudinales con una muestra más heterogénea, que abarcara otras culturas y regiones, ampliando el rango de edad a toda la etapa adolescente. En tercer lugar, dado que todos los instrumentos utilizados son auto-informados, habría que tener cuidado con el fenómeno de deseabilidad social. En este sentido, la utilización de otro tipo de pruebas heteroinformadas podría complementar los resultados obtenidos, profundizando en el conocimiento del ciberacoso y evitando el posible efecto de deseabilidad que puede darse en la utilización de los cuestionarios y escalas. Finalmente, puesto que el fenómeno del ciberacoso es un problema complejo en el que intervienen diferentes variables, podría considerarse el análisis de otros recursos y factores que ayuden a comprender esta situación y favorezcan su prevención.

\section{Implicaciones teóricas y prácticas}

A pesar de las limitaciones que presenta este estudio, los hallazgos obtenidos aportan una serie de importantes implicaciones, tanto a nivel teórico como práctico. Desde una perspectiva teórica, este estudio y sus resultados han intentado dar respuesta a algunas lagunas existentes en la literatura e incrementar el conocimiento acerca del papel de algunos factores y recursos personales que pueden ayudar y proteger frente a las situaciones de ciberacoso. Específicamente, se ha centrado en el papel conjunto de la gratitud y la IE sobre las conductas de ciberagresión. Igualmente, nuestros resultados permiten a los investigadores continuar profundizando en el análisis y desarrollo del papel de la gratitud y la IE en el ajuste psicológico de los adolescentes en situaciones de ciberacoso donde hay una escasez de estudios realizados.

Considerando una perspectiva más práctica, el factor protector de la gratitud sobre el ciberacoso y su potencial papel para compensar un menor desarrollo de las competencias emocionales podría ser considerado para ser introducido en los programas de prevención y reducción del ciberacoso. Así como las personas utilizan diferentes estrategias para afrontar las situaciones estresantes y las dificultades diarias, poder entrenar y desarrollar varios recursos personales facilitaría un mejor abordaje del complejo fenómeno del ciberacoso.

\section{Conclusión}

Este estudio pretendía profundizar en el conocimiento del papel conjunto de gratitud e IE en el contexto de ciberacoso. A pesar de que es necesaria mayor investigación, los resultados evidencian los beneficios de desarrollar y entrenar varias fortalezas y estrategias para proteger contra estas situaciones. Además, se corrobora y afianza la importancia de que los adolescentes desarrollen buenas habilidades emocionales para la prevención del ciberacoso. Del mismo modo, los hallazgos obtenidos aportan algunas evidencias sobre los beneficios de la práctica de la gratitud para combatir estas conductas, debido a su papel protector para disminuir los comportamientos de ciberagresión y a su posible apoyo o soporte en aquellos adolescentes que presenten un menor desarrollo de las competencias emocionales.

\section{Financiación}

Esta investigación ha sido financiada por el grupo PAIDI CTS-1048 (Junta de Andalucía) y el programa FEDER/ Junta de Andalucía (UMA 18-FEDERJA-147).

\section{Referencias}

Algoe, S.B., Fredrickson, B.L. y Gable, S.L. (2013). The Social Functions of the Emotion of Gratitude via Expression. Emotion, 13(4), 605-609. https:// doi.org/10.1037/a0032701

Antoniadou, N. y Kokkinos, C.M. (2015). Cyber and school bullying: Same or different phenomena? Aggression and Violent Behavior, 25, 363-372. https://doi.org/10.1016/j.avb.2015.09.013

Baroncelli, A. y Ciucci, E. (2014). Unique effects of different components of trait emotional intelligence in traditional bullying and cyberbullying. Journal of Adolescence, 37, 807-815. https://doi.org/10.1016/ j.adolescence.2014.05.009

Bono, G., Froh, J.J., Disabato, D., Blalock, D., McKnight, P. y Bausert, S. (2019). Gratitude's role in adolescent antisocial and prosocial behavior: A 4-year longitudinal investigation. The Journal of Positive Psychology, 14(1), 230-243. https:// doi.org/10.1080/17439760.2017.1402078

Calmaestra, J., Rodríguez-Hidalgo, A.J., Mero-Delgado, O. y Solera, E. (2020). Cyberbullying in adolescents from Ecuador and Spain: Prevalence and differences in gender, school year and ethnic-cultural background. Sustainability, 12(11), 4597. https:// doi.org/10.3390/su12114597

Camerini, A.L., Marciano, L., Carrara, A. y Schulz, P.J. (2020). Cyberbullying perpetration and victimization among children and adolescents: A systematic review of longitudinal studies. Telematics and Infor- 
Ciberacoso y recursos personales en adolescentes: las competencias emocionales y la gratitud como factores de protección ante las conductas de ciberagresión

matics, 49, 101362. https://doi.org/10.1016/ j.tele. 2020.101362

Cañas, E., Estévez, E., Martínez-Monteagudo, M.C. y Delgado, B. (2020). Emotional adjustment in victims and perpetrators of cyberbullying and traditional bullying. Social Psychology of Education, 0123456789. https://doi.org/10.1007/s11218-02009565-z

Cañas, E., Estévez, E., Marzo, J.C. y Piqueras, J.A. (2019). Psychological adjustment in cybervictims and cyberbullies in secondary education. Anales de Psicología / Annals of Psychology, 35(3), 434-443. https://doi.org/10.6018/analesps.35.3.323151

Carbonell, N. y Cerezo, F. (2019). El programa CIE: Intervención en ciberacoso escolar mediante el desarrollo de la Inteligencia Emocional. European Journal of Health Research, 5(1), 39. https://doi.org/10.30552/ ejhr.v5i1.136

Cejudo, J., Rodrigo-Ruiz, D., López-Delgado, M.L. y Losada, L. (2018). Emotional Intelligence and Its Relationship with Levels of Social Anxiety and Stress in Adolescents. International Journal of Environmental Research and Public Health, 15, 1073. https:// doi.org/10.3390/ijerph15061073

Chen, L., Wang, Y., Yang, H. y Sun, X. (2020). Emotional warmth and cyberbullying perpetration attitudes in college students: Mediation of trait gratitude and empathy. PLoS ONE, 15(7), 1-14. https:// doi.org/10.1371/journal.pone.0235477

Cho, Y. y Fast, N.J. (2012). Power, defensive denigration, and the assuaging effect of gratitude expression. Journal of Experimental Social Psychology, 48, 778 -782. https://doi.org/10.1016/j.jesp.2011.12.016

Declaración de Helsinki. (2013). Ethical Principles for Medical Research Involving Human Subjects. Journal of the American Medical Association, 310(20), 2191 -2194. https://doi.org/10.1001/jama.2013.281053

Deichert, N.T., Chicken, M.P. y Hodgman, L. (2019). Appreciation of Others buffers the associations of stressful life events with depressive and physical symptoms. Journal of Happiness Studies, 20(4), 1071-1088. https://doi.org/10.1007/s10902-0189988-9

Del Rey, R., Casas, J.A., Ortega-Ruiz, R., SchultzeKrumbholz, A., Scheithauer, H., Smith, P., Thompson, F., Barkoukis, V., Tsorbatzoudis, H., Brighi, A., Guarini, A., Pyżalski, J. y Plichta, P. (2015). Structural validation and cross-cultural robustness of the European Cyberbullying Intervention Project
Questionnaire. Computers in Human Behavior, 50, 141-147. https://doi.org/10.1016/ J.CHB.2015.03.065

Deng, Y., Xiang, R., Zhu, Y., Li, Y., Yu, S. y Liu, X. (2019). Counting blessings and sharing gratitude in a Chinese prisoner sample: Effects of gratitudebased interventions on subjective well-being and aggression. Journal of Positive Psychology, 14(3), 303-311. doi.org/10.1080/17439760.2018.1460687

DeWall, C.N., Lambert, N.M., Pond Jr., R.S., Kashdan, T.B. y Fincham, F.D. (2012). A grateful heart is a nonviolent heart: Cross-sectional, experience sampling, longitudinal, and experimental evidence. Social Psychological and Personality Science, 3(2), 232-240. https://doi.org/http:// doi.org/10.1177/1948550611416675

Estévez, E., Estévez, J.F., Segura, L. y Suárez, C. (2019). The influence of bullying and cyberbullying in the psychological adjustment of victims and aggressors in adolescence. International Journal of Environmental Research and Public Health, 16, 2080. https://doi.org/10.3390/ijerph16122080

Extremera, N. y Rey, L. (2016). Ability emotional intelligence and life satisfaction: Positive and negative affect as mediators. Personality and Individual Differences, 102, 98-101. https://doi.org/10.1016/ j.paid.2016.06.051

Extremera, N., Rey, L. y Sánchez-Álvarez, N. (2019). Validation of the Spanish version of the Wong Law Emotional Intelligence Scale (WLEIS-S). Psicothema, 31(1), 94-100. https://doi.org/10.7334/ psicothema2018.147

Garaigordobil, M. (2019). Prevención del cyberbullying: variables personales y familiares predictoras de ciberagresión. Revista de Psicología Clínica Con Niños y Adolescentes, 6(3), 9-17. https:// doi.org/10.21134/rpcna.2019.06.2.1

Garaigordobil, M. y Machimbarrena, J.M. (2019). Victimization and perpetration of bullying/cyberbullying: connections with emotional and behavioral problems and childhood stress. Psychosocial Intervention, 28(2), 67-73. https://doi.org/10.5093/pi2019a3

Garaigordobil, M. y Peña-Sarrionandia, A. (2015). Effects of an emotional intelligence program in variables related to the prevention of violence. Frontiers in Psychology, 6, 743. https://doi.org/10.3389/ fpsyg.2015.00743

García-Sancho, E., Salguero, J.M. y Fernández-Berrocal, P. 
(2014). Relationship between emotional intelligence and aggression: A systematic review. Aggression and Violent Behavior, 19(5), 584-591. https:// doi.org/10.1016/j.avb.2014.07.007

García-Vázquez, F.I., Parra-Pérez, L.G. y Valdés-Cuervo, A.A. (2020). The effects of forgiveness, gratitude, and self-control on reactive and proactive aggression in bullying. International Journal of Environmental Research and Public Health, 17(16), 5760. https://doi.org/10.3390/ijerph17165760

Geng, Y. (2018). Gratitude mediates the effect of emotional intelligence on subjective well-being: A structural equation modeling analysis. Journal of Health Psychology, 23(10), 1378-1386. https:// doi.org/10.1177/1359105316677295

Guse, T., Vescovelli, F. y Croxford, S.A. (2017). Subjective Well-Being and Gratitude Among South African Adolescents: Exploring Gender and Cultural Differences. Youth \& Society, 51(5), 591-615. https:// doi.org/https://doi.org/10.1177\% 2F0044118X17697237

Hayes, A.F. (2018). Introduction to Mediation, Moderation, and Conditional Process Analysis. A Regression-Based Approach ( ${ }^{a}$ ed.). Guilford Press.

Hinduja, S. y Patchin, J.W. (2019). Connecting Adolescent Suicide to the Severity of Bullying and Cyberbullying. Journal of School Violence, 18(3), 333346. https:// doi.org/10.1080/15388220.2018.1492417

Howells, K. (2014). An exploration of the role of gratitude in enhancing teacher-student relationships. Teaching and Teacher Education, 42, 58-67. https:// doi.org/10.1016/j.tate.2014.04.004

Howells, K., Stafford, K., Guijt, R. y Breadmore, M. (2017). The role of gratitude in enhancing the relationship between doctoral research students and their supervisors. Teaching in Higher Education, 22 (6), 621-638. https:// doi.org/10.1080/13562517.2016.1273212

Inglés, C.J., Torregrosa, M.S., García-Fernández, J.M., Martínez-Monteagudo, M.C., Estévez, E. y Delgado, B. (2014). Conducta agresiva e inteligencia emocional en la adolescencia. European Journal of Education and Psychology, 7(1), 29-41. https:// doi.org/10.30552/ejep.v7i1.97

Iranzo, B., Ortega-Barón, J., Carrascosa, I. y Clemente, A.J. (2019). Ajuste psicosocial de adolescentes agresores a través del teléfono móvil y de internet. International Journal of Developmental and Educa- tional Psychology. Revista INFAD de Psicología., 1 (1), 79-92. https://doi.org/10.17060/ ijodaep.2019.n1.v1.1387

Jans-Beken, L., Lataster, J., Peels, D., Lechner, L. y Jacobs, N. (2018). Gratitude, Psychopathology and Subjective Well-Being: Results from a 7.5-Month Prospective General Population Study. Journal of Happiness Studies, 19(6), 1673-1689. https:// doi.org/10.1007/s10902-017-9893-7

Kokkinos, C.M. y Voulgaridou, I. (2017). Relational and cyber aggression among adolescents: Personality and emotion regulation as moderators. Computers in Human Behavior, 68, 528-537. https:// doi.org/10.1016/j.chb.2016.11.046

Kowalski, R.M., Limber, S.P. y McCord, A. (2019). A developmental approach to cyberbullying: Prevalence and protective factors. Aggression and Violent Behavior, 45, 20-32. https://doi.org/10.1016/ j.avb.2018.02.009

Lavelock, C.R., Griffin, B.J., Worthington, E.L., Benotsch, E.G., Lin, Y., Greer, C.L., Garthe, R.C., Coleman, J.A., Hughes, C.M., Davis, D.E. y Hook, J.N. (2016). A Qualitative Review and Integrative Model of Gratitude and Physical Health. Journal of Psychology and Theology, 44(1), 55-86. https:// doi.org/10.1177/009164711604400105

Lin, C.-C. (2015). The relationships among gratitude, selfesteem, depression, and suicidal ideation among undergraduate students. Scandinavian Journal of Psychology, 56(6), 700-707. https:// doi.org/10.1111/sjop.12252

Lozano-Blasco, R., Cortés-Pascual, A. y Latorre-Martínez, M.P. (2020). Being a cybervictim and a cyberbully - The duality of cyberbullying: A meta-analysis. Computers in Human Behavior, 111, 106444. https://doi.org/10.1016/j.chb.2020.106444

Martínez-Monteagudo, M.C., Delgado, B., GarcíaFernández, J.M. y Rubio, E. (2019). Cyberbullying, Aggressiveness, and Emotional Intelligence in Adolescence. International Journal of Environmental Research and Public Health, 16(24). https:// doi.org/10.3390/ijerph16245079

Mayer, J.D., Caruso, D.R. y Salovey, P. (2016). The Ability Model of Emotional Intelligence: Principles and Updates. Emotion Review, 8(4), 290-300. https:// doi.org/10.1177/1754073916639667

Mayer, J.D. y Salovey, P. (1997). What is emotional intelligence? En P. Salovey y D. J. Sluyter (Eds.), Emotional development and emotional intelligence: Educa- 
Ciberacoso y recursos personales en adolescentes: las competencias emocionales y la gratitud como factores de protección ante las conductas de ciberagresión

tional implications (pp. 528-549). Harper Collins.

McCullough, M.E., Emmons, R.A. y Tsang, J.-A. (2002). The Grateful Disposition: A Conceptual and Empirical Topography. Journal of Personality and Social Psychology, 82(1), 112-127. https:// doi.org/10.1037/0022-3514.82.1.112

Morales Rodríguez, F.M. (2017). Relaciones entre afrontamiento del estrés cotidiano, autoconcepto, habilidades sociales e inteligencia emocional. European Journal of Education and Psychology, 10(2), 41-48. https://doi.org/10.1016/j.ejeps.2017.04.001

Ortega-Ruiz, R., Del Rey, R. y Casas, J.A. (2016). Evaluar el bullying y el cyberbullying validación española del EBIP-Q y del ECIP-Q. Psicología Educativa, 22 (1), 71-79. https://doi.org/10.1016/ j.pse.2016.01.004

Preś, J.E., Kurpisz, J., Pełka-Wysiecka, J., Adamowicz, K., Suchecka-Fidura, D., Ilnicka, M., Mak, M. y Samochowiec, J. (2020). What Regulates Gratitude Response of Women and Men? The Role of the Received Good, Psychosocial Factors, and Repayment. Psychological Reports, 123, 395-419. https:// doi.org/10.1177/0033294118811620

Quintana-Orts, C. y Rey, L. (2018). Forgiveness and cyberbullying in adolescence: Does willingness to forgive help minimize the risk of becoming a cyberbully? Computers in Human Behavior, 81, 209-214. https://doi.org/10.1016/j.chb.2017.12.021

Rey, L., Sánchez-Álvarez, N. y Extremera, N. (2018). Spanish Gratitude Questionnaire: Psychometric properties in adolescents and relationships with negative and positive psychological outcomes. Personality and Individual Differences, 135, 173-175. https:// doi.org/10.1016/j.paid.2018.07.005

Salve, S. y Lavalekar, A. (2017). Role of Dispositional Gratitude and Authenticity in Emotional Intelligence among Late Adolescent Boys and Girls. Indian Journal of Positive Psychology, 8(3), 374. https:// doi.org/10.15614/ijpp/2017/v8i3/161986

Sanjuán, C. (2019). Violencia Viral, análisis de la violencia contra la infancia y la adolescencia en el entorno digital. https://www.savethechildren.es/sites/default/ files/imce/docs/informe_violencia_viral_1.pdf

Schoeps, K., Villanueva, L., Prado-Gascó, V.J. y MontoyaCastilla, I. (2018). Development of emotional skills in adolescents to prevent cyberbullying and improve subjective well-being. Frontiers in Psychology, 9, 2050. https://doi.org/10.3389/fpsyg.2018.02050 emotional intelligence in adolescent cyberaggressors and cybervictims. International Journal of Environmental Research and Public Health, 17(13), 1-14. https://doi.org/10.3390/ijerph17134681

Smith, P.K., Mahdavi, J., Carvalho, M., Fisher, S., Russell, S. y Tippett, N. (2008). Cyberbullying: Its nature and impact in secondary school pupils. Journal of Child Psychology and Psychiatry and Allied Disciplines, 49(4), 376-385. https://doi.org/10.1111/ j.1469-7610.2007.01846.x

Tokunaga, R.S. (2010). Following you home from school: A critical review and synthesis of research on cyberbullying victimization. Computers in Human Behavior, 26(3), 277-287. https://doi.org/10.1016/ j.chb.2009.11.014

Wong, C.-S. y Law, K.S. (2002). The effects of leader and follower emotional intelligence on performance and attitude: An exploratory study. The Leadership Quarterly, 13(3), 243-274. https://doi.org/10.1016/ S1048-9843(02)00099-1

Wood, A.M., Froh, J.J. y Geraghty, A.W.A. (2010). Gratitude and well-being: A review and theoretical integration. Clinical Psychology Review, 30(7), 890905. $\quad$ https://doi.org/https://doi.org/10.1016/ j.cpr.2010.03.005

Yost-Dubrow, R. y Dunham, Y. (2018). Evidence for a relationship between trait gratitude and prosocial behaviour. Cognition and Emotion, 32(2), 397-403. https://doi.org/10.1080/02699931.2017.1289153

You, S., Lee, J. y Lee, Y. (2020). Relationships between gratitude, social support, and prosocial and problem behaviors. Current Psychology. https://doi.org/ https://doi.org/10.1007/s12144-020-00775-4

You, S. y Lim, S.A. (2016). Longitudinal predictors of cyberbullying perpetration: Evidence from Korean middle school students. Personality and Individual Differences, 89, 172-176. https://doi.org/10.1016/ j.paid.2015.10.019

Yudes, C., Rey, L. y Extremera, N. (2020). Predictive Factors of Cyberbullying Perpetration amongst Spanish Adolescents. International Journal of Environmental Research and Public Health, 17(11), 3967. https://doi.org/10.3390/ijerph17113967

Zych, I., Farrington, D.P. y Ttofi, M.M. (2019). Protective factors against bullying and cyberbullying: A systematic review of meta-analyses. Aggression and Violent Behavior, 45, 4-19. https://doi.org/10.1016/ j.avb.2018.06.008

Segura, L., Estévez, J.F. y Estévez, E. (2020). Empathy and 